City University of New York (CUNY)

CUNY Academic Works

\title{
Mapping Gadolinium Contrast in a Complex Ionic and Photosynthesis Environment of Pineapple by Near-infrared and X- ray Imaging
}

Subhendra Sarkar

CUNY New York City College of Technology

Zoya Vinokur

CUNY New York City College of Technology

Chen Xu

CUNY New York City College of Technology

Tetiana Soloviova

CUNY New York City College of Technology

Amina Shahbaz

CUNY New York City College of Technology

See next page for additional authors

\section{How does access to this work benefit you? Let us know!}

More information about this work at: https://academicworks.cuny.edu/ny_pubs/620

Discover additional works at: https://academicworks.cuny.edu

This work is made publicly available by the City University of New York (CUNY).

Contact: AcademicWorks@cuny.edu 
Authors

Subhendra Sarkar, Zoya Vinokur, Chen Xu, Tetiana Soloviova, Amina Shahbaz, and Aldona Gjoni 
Mapping Gadolinium Contrast in a Complex Ionic and Photosynthesis Environment of Pineapple by Near-infrared and X-ray Imaging

Subhendra Sarkar ${ }^{*}$, Zoya Vinokur ${ }^{1}$, Chen $\mathrm{Xu}^{2}$, Tetiana Soloviova ${ }^{1}$, Amina Shahbaz ${ }^{1}$, Aldona Gjoni ${ }^{1}$.

Departments of ${ }^{1}$ Radiologic Technology \& Medical Imaging, and ${ }^{2}$ Computer Engineering Technology, New York City College of Technology, City University of New York, New York, USA.

*Corresponding Author: Subhendra N. Sarkar （ORCID ID: 0000-0002-5942-2801) New York City College of Technology/CUNY Dept of Radiologic Technology \& Medical Imaging 300 Jay St, A-414 Brooklyn, NY 11201

Email: ssarkar@CityTech.CUNY.Edu

Journal Submitted: $\quad$ Spectroscopy Letters

Short Title:

Gadolinium Complex in Pineapple: Diffusion and Photosynthesis Study by NIR and X-Ray Imaging

Keywords: $\quad$ Gadolinium Contrast, Near infrared spectroscopy, Diffusivity, Dark Photosynthesis, X Ray of Pineapple

Word count: 3366

Conflict of Interest: None of the authors have any conflict of interest 


\title{
Mapping Gadolinium Contrast in a Complex Ionic and Photosynthesis Environment of Pineapple by Near-infrared and X-ray Imaging
}

\begin{abstract}
This work explores the diffusivity of a lanthanide complex, Eovist (Gadolinium-Ethoxy Benzyl

Diethylenetriamine pentaacetate) that is stable in neutral media but is not in acidic environment. In the current work an acidic fruit model like pineapple that is rich in transition metals was used and a possible transmetallation reaction among Eovist and transition metal complexes was tested using x-ray imaging. Another goal of this work was to perturb the usual and the unusual photosynthesis systems that pineapple has maintained for millions of years during the evolution of circadian genes for efficient water conservation by dark photosynthesis. To detect such photosynthesis Near infrared reflection spectroscopy was used for pineapple samples in a dark box, and to test if added lanthanide complex and pre-existing transition metals in pineapple can affect such photosynthesis systems, X-ray imaging and Near infrared reflection spectroscopy were performed pre and post infusion of Eovist in pineapple samples. Time series, low energy Xray images showed heterogeneous distribution of Gadolinium at different locations in the pineapple samples. This could be explained if competing transition metal ions could steal Diethylenetriamine pentaacetate ligands from the inner sphere of Gadolinium altering the size and thus diffusivity of Gadolinium complex. Near infrared data showed red-shifted peaks characteristic of dark photosynthesis, similar to ones observed in cyano bacteria, but did not show any noticeable shifts due to the infused Gadolinium complex. Transmetallation and ionic exchange in such unique model fruits may have scientific, medical and agricultural implications for efficient solar cells, tissue regeneration as well as enhanced plantation using high efficiency photosynthesis.
\end{abstract}




\section{Introduction}

Pineapple has several minerals at moderately high concentrations that are mainly $\mathrm{K}, \mathrm{Mg}$ and $\mathrm{Ca}$, with $\mathrm{Mg}$ and $\mathrm{Ca}$ an order of magnitude less than $\mathrm{K}$ while four more minerals, $\mathrm{Fe}, \mathrm{Mn}, \mathrm{Zn}$ and $\mathrm{Cu}$ are at one order of magnitude lower concentration than $\mathrm{Ca}$ and $\mathrm{Mg}$. These minerals control significant biological activities. ${ }^{[1,2]} \mathrm{Mg}$ is utilized by many enzymes, especially from the sugar and protein kinase families to catalyze ATP (Adenosine Tri Phosphate) dependent phosphorylations. It is important to note that the $\mathrm{pH}$ and water content of pineapple are in the range of 3.5-4.1 and approx $87 \%$, resulting in a mixture of richly hydrated cations and others with partial hydrations.

Gadolinium based contrast agents were considered extremely safe and without major adverse effects on human tissue. Recently with better understanding of Gd chemistry, tissue deposition has been conclusively demonstrated in brain as well as in bone and skin. This occurs with less stable group 1 linear Gd ligands but recent postmortem studies now confirm that Gd deposition also occurs with all Gd contrast agents that lose the DTPA (Diethylenetriamine pentaacetate) ligands baring Gd ion. ${ }^{[3]}$ To our knowledge, the competing role of alkali and transition metal ions has not been studied for Gd agents.

This is the first work that tests this competition by infusing a relevant $\mathrm{Gd}^{3+}$ complex with biphasic behavior in human tissue (early and delayed absorption in liver cells) and apply imaging to monitor the time dependent Gd distribution inside a complex ionic medium like pineapple. Note that $\mathrm{Gd}^{3+}$ coordination chemistry is much less predictable than transition metals due to the 
absence of well-studied ligand fields and due to the larger coordination number of 8-10. The ionic radius of $\mathrm{Gd}^{3+}$ is $0.99 \AA$, similar to divalent $\mathrm{Ca}^{2+} \cdot{ }^{[4]}$ If $\mathrm{Gd}^{3+}$ becomes fully hydrated, it is supposed to occupy the center of a square antiprism with 8 water molecules at the corners of this inner sphere with a residence time of approximatelyl ns for each water molecule during which its electron lone pair engages with $\mathrm{Gd}^{3+}$ ion.

Most Gd-DTPA complexes in biomedicine have one ligand available for water. Gd-EOB (Gadolinium-Ethoxy Benzyl) DTPA was chosen since it is an amphipathic derivative of GdDTPA (Gd-DTPA with a covalently bound lipophilic ethoxybenzyl arm, Fig 1). If it can be partially freed from some of the DTPA ligands, we believe the mobile $\mathrm{Gd}^{3+}$ complex may then compete with transition metal complexes of $\mathrm{Ca}^{2+}$, for example. This may affect those reactions that require $\mathrm{Ca}^{2+}$ for proper function and, in doing so, the trivalent ion $\mathrm{Gd}^{3+}$ binds with much higher affinity than $\mathrm{Ca}^{2+}$. When a $\mathrm{Ca}^{2+}$ binding enzyme is involved, lanthanide ion replacement can alter the kinetics of the biological process catalyzed by that enzyme and may be beneficial to treat suitable disease process like osteo-arthritis. ${ }^{[5]}$

It has been shown, ${ }^{[5]}$ that osteoporotic fracture patients exposed to Gd have significantly lower Gd concentrations than osteoarthritis patients indicating different mechanisms of metal incorporation and retention in osteoporotic bone tissues, and may initiate greater endogenous Gd release for patients with increased rates of bone resorption (e.g. osteoporosis patients as well as menopausal, pregnant, and lactating women) who need Gd-based DTPA contrast media during their Radiological diagnostics. 
Magnetic Resonance Imaging (MRI) contrast Eovist is an ideal candidate with $\mathrm{Gd}^{3+}$ ion loosely bound to other negative structures like phenol rings. Such complexes can supply or absorb both protons and electrons and in that process may alter key biochemical pathways. We were able to observe spectral peaks that have been implicated as Near infrared (NIR) driven photosynthesis at precise absorption wavelengths in cut pineapple. Our research aims to explore if at the low levels of $\mathrm{pH}$ in pineapple lanthanide complex like Eovist (Gadolinium-DTPA complex) may change the inner coordination sphere and show higher or more restricted diffusivity. We used both NIR as well as medical imaging tools to map out the Gd dynamics.

\begin{abstract}
Another important goal was to perturb the usual and the unusual photosynthesis that pineapple has maintained for millions of years during plant evolution. ${ }^{[7,8]}$ Delayed NIR spectroscopy on Gadolinium infused pineapple was performed from various cut samples to investigate effects on those NIR peaks as a marker of altered electron dynamics during photosynthesis. NIR induced photosynthesis of pineapple ${ }^{[9]}$ requires careful sampling of the fruit to avoid disturbing the original respiration rate ${ }^{[10]}$ and has been ill understood.
\end{abstract}

\title{
Materials and Methods
}

$\underline{\text { Structure and Properties of Gadolinium complex in acidic environment }}$

Gadolinium-Ethoxy Benzyl DTPA (Gd-EOB-DTPA, Eovist, Bayer Pharmaceuticals), shown in Figure 1, was chosen since it is an amphipathic derivative of Gd-DTPA (Gd-DTPA with a covalently bound lipophilic ethoxybenzyl group) with a higher protein binding capacity than 
other Gd-DTPA, $10 \%$ vs. $1.5 \%$ ). ${ }^{[11]}$ In addition, Gd-EOB-DTPA, being an open-chain

Gadolinium chelate, has a lower kinetic stability than macrocyclic gadolinium chelates. ${ }^{[12]}$

\section{Figure 1 here.}

Fresh, intact pineapples were imaged by MRI systems to identify proton spin diffusivity at various locations using diffusion tensor imaging (unpublished work in progress at our collaborating laboratory). Water diffusion in the absence of Eovist seems to be uniform throughout fresh pineapple. Those pre-screened pineapple samples were selected for Eovist infusion to study diffusion by NIR and X-ray imaging in the current work.

Two cm thick slices containing Eovist were carefully sliced by sharp knife to avoid blunt trauma affecting pineapple respiration rate ${ }^{[10]}$ and were imaged by low $\mathrm{kV}$ X-ray. The imaging technique was optimized to be $45 \mathrm{kVp}$ and $4.7 \mathrm{mAs}$ (focal spot size, $0.25 \mathrm{~mm}$ ) at 40" SID using a digital X-ray system from Quantum Medical, Ronkonkoma, NY. Gd complex used seems to have absorbed X-rays by the L-edge X-ray absorption modes instead of K-edges since our effective $\mathrm{kV}$ was much less than $45 \mathrm{kVp}$.

The pineapple slices were cut in $2 \times 2 \mathrm{~cm}$ squares just prior to NIR spectroscopy acquisition from Eovist infused and Eovist free areas using an AvaSpec-ULS2048 system in reflection mode in the wavelength range from 400 to $1000 \mathrm{~nm}$, and resolution of $0.6 \mathrm{~nm}$ with 200 scan averaging. The samples were studied in a dark box to avoid ambient light allowing only NIR to induce photosynthesis by the dark photosynthesis mechanism available in pineapple. ${ }^{[7,8]}$ 


\section{Results}

$\underline{\text { X-ray imaging of Gadolinium diffusivity }}$

K-edge for $\mathrm{Gd}$ is $50 \mathrm{kV}$ which was outside the peak output of our $45 \mathrm{kVp} \mathrm{X}$-ray and was not utilized. However, the effective $\mathrm{kV}$ was approximately $15-20$ in our $45 \mathrm{kVp}$ beam and hence was quite efficient to be absorbed by Gd L-edges, spanning 5-8 $\mathrm{kV}$ that resulted in the absorption spots in Figure 2 shown below.

Figure 2 shows both slow as well as fast spread of Eovist. The diffusivity at the core was not measured. Even the slow spread near the rinds seems to produce three different diffusivities. Two locations shown here as examples (1 and 2 O'clock positions, arrows) where Eovist was infused demonstrate how the diffusion rates were compared along major and minor axes for the spots. By comparing these two slices, A\&C panels at the left with $\mathrm{B} \& \mathrm{D}$ at the right one can argue that Eovist structures are intact next to the rinds where $\mathrm{pH}$ seems to be high. However, at the core for the slice cut from upper part of the pineapple (image slice A at the top) Gd spreads within minutes presumably due to free Gadolinium after losing one or more DTPA ligands at low $\mathrm{pH}$.

\section{Figure 2 here.}

The diffusivities of several elliptical spots are plotted in Figure 3(A) and 3(B). The central slice shows more structured, radial uptake of Gd while the upper slice has fast, heterogeneous Gd 
uptake throughout the slice. This is indicative of slow, structured diffusion at the central slice and fast, heterogeneous diffusivity at top slice. From our collaborating Magnetic Resonance research laboratory we had observed that the diffusivity of water is quite uniform all throughout our fresh pineapple samples as per diffusion MRI prior to Eovist infusions. Diffusion MRI results of various Gd-DTPA contrast agents in fruits are under progress in high field MRI systems and are not reported here.

The $\mathrm{pH}$ distribution in the pineapple is heterogeneous allowing perhaps different divalent and trivalent transition metals $\left(\mathrm{Ca}^{2+}, \mathrm{Mg}^{2+}\right.$ that are abundant, and possibly $\mathrm{Fe}^{3+}$ that has lower concentration than prior two) to partially remove one or more DTPA moieties from central Gd in Eovist antiprism structure allowing more water molecules to rush to the inner sphere. This seems to happen at the core in superior slice of the pineapple showing Gd "flood" at the core within minutes. However, slightly away from the rim, some spots have the higher diffusivity values as shown in the fast groups in the top right corners of Figure 3.

\section{Figure 3(A, Left) and 3(B, Right) here}

$\underline{\text { Near Infra Red Spectroscopy Results }}$

In our model system we observed two peaks that have been observed in cyano bacteria induced photosynthesis ${ }^{[9]}$ and seem to confirm the photosynthesis genes (Crassulacean acid metabolism genes or CAM) extracted in pineapple ${ }^{[7,8]}$ and in our system seem to have resulted from photosynthesis induced by NIR photons (weak one as $C h l d$ and stronger one from $C h l f$ photosynthesis centers respectively). We observed slightly shifted peaks for pineapple, 640 and 
$680 \mathrm{~nm}$ (Figure 4) both of which are red-shifted by $30 \mathrm{~nm}$ compared to cyano bacteria induced photosynthesis. ${ }^{[9]}$ However, there does not seem to be any noticeable effect due to infused Gadolinium complex on NIR spectrum and may indicate insufficient Gd infusion or postinfusion delay was outside the temporal resolution of the photosynthesis reactions.

\section{Figure 4 here}

\section{$\underline{\text { Discussion }}$}

Pineapple has several minerals at high as well as at low concentrations that can compete with Gd complexes in aqueous solution. However, their $\mathrm{pH}$ dependence is not well established when high coordination numbers are involved as in Eovist. Of particular interest for us are $\mathrm{Ca}, \mathrm{Mg}, \mathrm{Cu}, \mathrm{Mn}$ and Fe that are relevant in significant biological activities. ${ }^{[1,2]}$ This work demonstrates the slow diffusion of Gd dye near the rind and structured as well as diffuse uptake of dye in different heights of the pineapple. This is important and pineapple can be used as a model biological system to test transmetallation and ligand exchange between several transition metal ions and lanthanide complexes like Gd-DTPA that are of significant biomedical interests.

The typical ligands in Eovist are linear amines and provide 8 donor atoms such that the $9^{\text {th }}$ candidate could be a single water molecule in the inner sphere of $\mathrm{Gd}^{3+}$. This geometry allows the bulk water matrix to exchange more water molecules through this single arm and allows sharing lone pair electrons to compensate for the electronic deficiency of $\mathrm{Gd}^{3+}$. We may estimate it to be approximately $1 \mathrm{~ns}$ for each donor water based on other Gd-DTPA systems ${ }^{[13]}$, which is two 
orders of magnitude slower than the symmetrical ligand situations with eight water molecules in the inner coordination sphere, $\mathrm{Gd}(\mathrm{H} 2 \mathrm{O})_{8}{ }^{3+}$.

In acidic environment of pineapple there may be the following kinetics at work for Eovist:

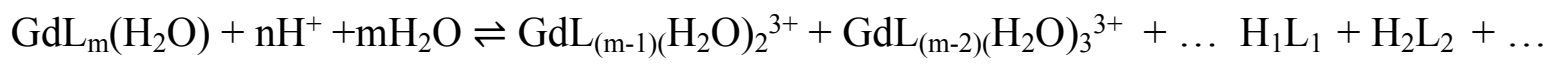

In the presence of transition metal ions $\left(\mathrm{M}^{+2}\right)$ some of the $\mathrm{H}_{\mathrm{i}} \mathrm{L}_{\mathrm{i}}$ species are replaced by $\mathrm{ML}_{2}$. The enthalpy of $\mathrm{ML}_{2}$ formation is quite variable and is available in the literature. We feel $\mathrm{Cu}^{2+}$ and $\mathrm{Mn}^{2+}$ may favor Eovist break up, as will $\mathrm{Fe}^{3+}$ since the order of affinity to compete with $\mathrm{Gd}^{3+}$ for these ions present is $\mathrm{Fe}^{3+}>\mathrm{Cu}^{2+}>\mathrm{Zn}^{2+}$. Note that although $\mathrm{Mg}$ and $\mathrm{Ca}$ are present at an order of magnitude higher concentration than $\mathrm{Fe}$ and $\mathrm{Cu}$, the divalent $\mathrm{Ca}$ and $\mathrm{Mg}$ form weak complexes and may not transmetallate $\mathrm{Gd}$.

$\mathrm{Gd}^{3+}$ coordination chemistry is difficult to understand although it is very important for accuracy in biomedical diagnostic applications. At low $\mathrm{pH}$ we believe $\mathrm{Gd}^{3+}$ complex may open up, release one or more DTPA ligand and can successfully diffuse into several bioreaction pathways that otherwise utilize transition metal ions. The altered Gd complex with less DTPA arms may be more mobile as we have observed at the core of our pineapple model. We believe $\mathrm{Fe}^{3+}$ and $\mathrm{Cu}^{2+}$ are mainly responsible for fast diffusion of $\mathrm{Gd}^{3+}$ in our X-ray images with possible breakdown of Eovist complex. This seems to indicate that in the strong acidic region (significant contribution of $\mathrm{nH}^{+}$) we expect Eovist complex to partially break down and create more aqua Gd complexes that are much smaller and thus more efficient to disrupt biochemical reactions involving transition metals. 
For prospective applications of our work one may note that different metal ions are integral components of bone tissue, playing functional roles in the physiological cellular environment as well as in the course of bone healing. ${ }^{[14,15]}$ To expedite bone resorption during post-trauma rehabilitation one may substitute $\mathrm{Ca}$ sites with suitable lanthanide ions that have higher affinity for enzymes than the current choice of $\mathrm{Sr}^{2+}$ in hydroxyapatite.

In our model pineapple system we observed similar peaks that were slightly red-shifted than those observed in cyano bacteria induced photosynthesis perhaps due to other protonated species including transition metal ions present in the photosynthesis compartments environment compared to the bacterial habitats. ${ }^{[9]}$

If one can control the dark and light photosynthesis of such special plants a novel second application would be efficient energy conversion by bioactive solar panel operation both during day and night in wetlands as well as in dry regions of globe since the novel (CAM) photosynthesis pathways conserve water and avoid high respiration rates common during the daytime. Hence, we believe, this report on fast and slow diffusing $\mathrm{Gd}^{3+}$ mapping in pineapple and its role in affecting various biochemical processes may stimulate interest and open choices to use lanthanide complexes for rehabilitation biomaterials as well as in energy sector.

\section{Declaration of Interest Statement}

Authors declare no financial interest or other conflicts of interest while conducting this research. 


\section{Acknowledgments}

We would like to acknowledge help from Jody-Ann Douglas for troubleshooting assistance. This work was made possible by undergraduate research scholar program (CRSP) stipends to TS, AS and AG during the academic year 2019-2020. 


\section{References}

[1] Sopie Salomé, Y. E.; Laurent, K. K.; Pierre Irénée, B. J.; Patrice, K.; Hilaire, K. T.

Comparison of Pineapple Fruit Characteristics of Plants Propagated in Three Different Ways: By Suckers, Micropropagation and Somatic Embryogenesis. J. Nutr. Food Sci., 2011, 1, 110.

[2] Lu, X-H.; Sun, D-Q.; Wu, Q-S.; Liu, S-H.; Sun, G-M. Physico-Chemical Properties, Antioxidant Activity and Mineral Contents of Pineapple Genotypes Grown in China. Molecules, 2014, 19, 8518-8532.

[3] Murata, K.; Gonzalez-Cuyar, L. F.; Maravilla, K. R. Gadolinium tissue deposition in brain and bone. Magnetic Reson. Imaging, 2016, 34, 1359-1365.

[4] Sherry, A. D.; Caravan, P.; Lenkinski, R. E. A primer on gadolinium chemistry. J Magn Reson Imaging, 2009, 30, 1240-1248.

[5] Darnall, D. W.; Birnbaum, E. R. Lanthanide ions activate alpha-amylase. Biochemistry, 1973, 12, 3489-3491.

[6] Darrah, T. H.; Prutsman-Pfeiffer, J. J.; Poreda, R. J.; Campbell, M. E.; Hauschka, P. V.; Hannigan, R. E.' Incorporation of excess gadolinium into human bone from medical contrast Agents. Metallomics, 2009, 1, 479-488.

[7] Bai, Y.; Dai, X.; Li, Y.; Wang, L.; Li, W.; Liu, Y.; Cheng, Y.; Qin, Y. Identification and characterization of pineapple leaf lncRNAs in crassulacean acid metabolism (CAM) photosynthesis pathway. Scientific Rep., 2019, 9, 6658.

[8] Ming, R.; VanBuren, R.; Wai, C. M.; Tang, H.; Schatz, M. C.; Bowers, J. E.; Lyons, E.; Wang, M-L.; Chen, J.; Biggers, E.; et al. The pineapple genome and the evolution of CAM photosynthesis. Nat. Genet., 2015, 47, 1435-1442. 
[9] Ku“ hl, M.; Trampe, E.; Mosshammer, M.; Johnson, M.; Larkum, A. W.; Frigaard, NU.; Koren, K. Substantial near-infrared radiation-driven photosynthesis of chlorophyll fcontaining cyanobacteria in a natural habitat. eLife, 2020, 9, e50871.

[10] Finnegan, E.; Mahakam, P. V.; O’Connell, M.; Francis, G. A.; O’Beirne, D. Modeling respiration in fresh-cut pineapple and prediction of gas permeability needs for optimal modified atmosphere packaging. Postharvest Biol.Technol., 2013, 79, 47-53.

[11] Van Beers, B. E.; Pastor, C. M.; Hussain, H. K. Primovist, Eovist: What to expect? J. Hepatol., 2012, 57, 421-429.

[12] Port, M.; Idee, J. M.; Medina, C.; Robic, C.; Sabatou, M.; Corot, C. Efficiency, thermodynamic and kinetic stability of marketed gadolinium chelates and their possible clinical consequences: a critical review. Biometals, 2008, 21, 469-490.

[13] Micskei, K.; Helm, L.; Brucher, E.; Merbach, A. E. Oxygen-17 NMR study of water exchange on gadolinium polyaminopolyacetates $[\mathrm{Gd}(\mathrm{DTPA})(\mathrm{H} 2 \mathrm{O})] 2$ - and [Gd(DOTA)(H2O)]- related to NMR imaging. Inorg. Chem., 1993, 32, 3844-3850. [14] Glenske, K.; Donkiewicz, P.; Köwitsch, A.; Milosevic-Oljaca, N.; Barbeck, M. Applications of Metals for Bone Regeneration. Materials, 2018, 19, 826. [15] Matsunaga, K. First-principles study of substitutional magnesium and zinc in hydroxyapatite and octacalcium phosphate. J. Chem. Phys., 2008, 128, 245101. 


\section{Figure Captions}

Figure 1. Structure of Gd- ethoxybenzyl DTPA (Diethylenetriamine pentaacetate) (Eovist, Bayer Pharmaceuticals) that was used in the diffusion experiments here. At low $\mathrm{pH}$ in pineapple Eovist may lose some of the DTPA ligands, particularly in the presence of competing transition metal ions.

Figure 2. Low energy $\mathrm{X}$ ray image, from L-edge X-ray absorption by $\mathrm{Gd}^{3+}$, of slowly diffusing Eovist from an upper slice 2 hours apart (Top, A\&B) and the central slice 2 hours apart (Bottom, C\&D) of pineapple samples showing two locations as examples (1 and 2 O'clock positions, arrows) where Eovist was infused but seems to diffuse very slowly during the $2 \mathrm{hr}$ observation periods. By comparing these two slices, $A \& C$ panels at the left with $B \& D$ at the right one can argue that Eovist structures are intact next to the rinds where $\mathrm{pH}$ may be high. However, at the core for the upper part of pineapple (image slice A at the top) Gd spreads within minutes presumably due to free Gadolinium with one or more DTPA ligands stripped.

Figure 3 (A, Left), 3 (B, Right). Measured diffusivities along the major and minor axes of elliptical Eovist spots are shown and grouped as slow, medium and fast groups (average diffusivities are noted in the plots, ranging an order of magnitude) presumably indicating transmetallation of $\mathrm{Gd}^{3+}$ with $\mathrm{Ca}^{2+}$ and $\mathrm{Mg}^{+2}$ ions or other mechanisms to free $\mathrm{Gd}^{3+}$ ions that explains faster diffusion of $\mathrm{Gd}^{3+}$ with smaller inner sphere than the original Eovist.

Figure 4. Near infrared background spectrum of the dark box (bottom trace, gray) that was subtracted from near infrared peaks of cut pineapple samples infused with Eovist (middle trace, black) and without Eovist (top trace, gray), Both the samples show near infrared induced photosynthesis peaks at 640 and $680 \mathrm{~nm}$ for pineapple systems, red-shifted by $30 \mathrm{~nm}$ compared to photosynthesis peaks (approximately 670 and $810 \mathrm{~nm}$ ) observed in cyano bacteria. [9] 


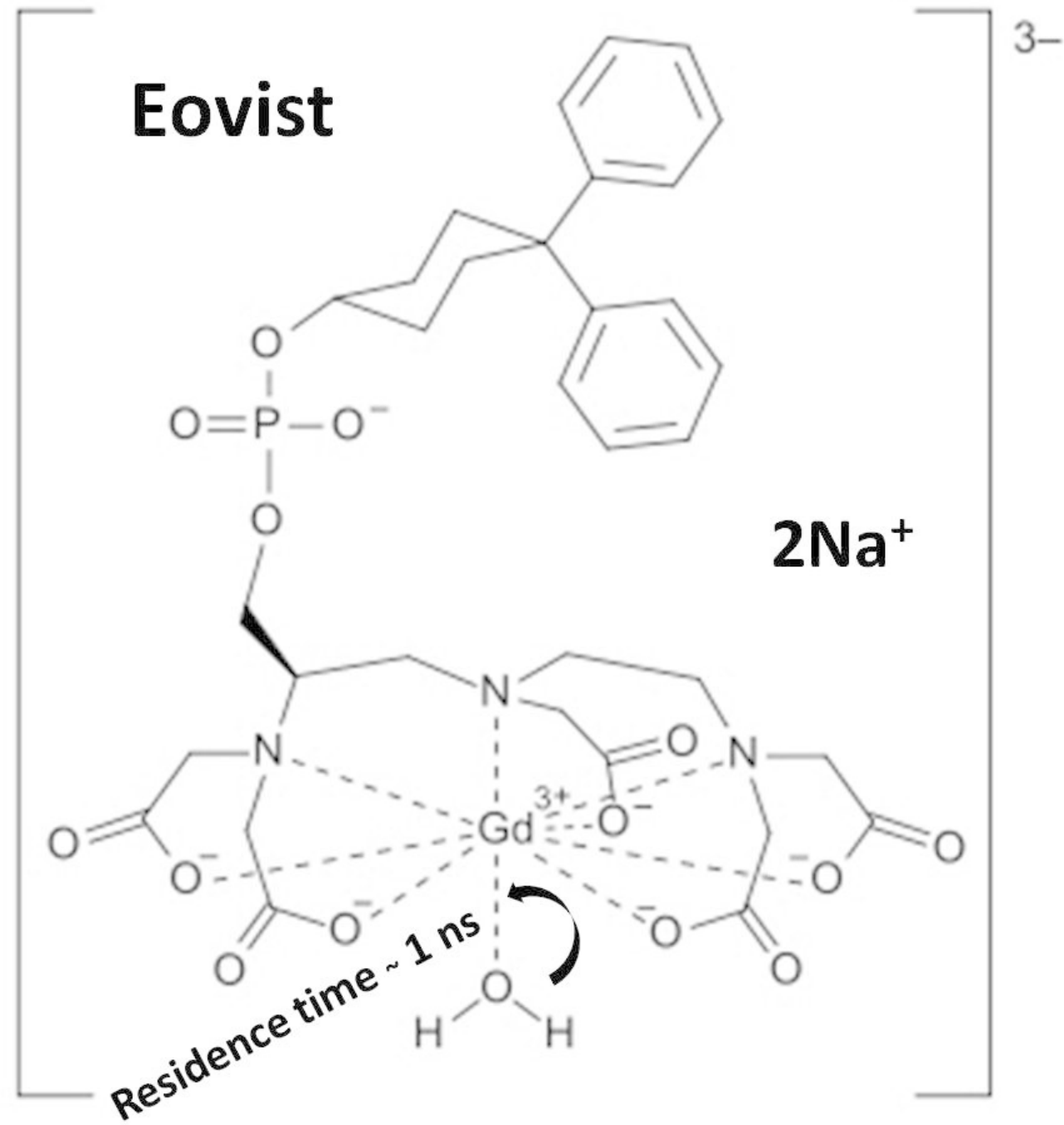

Figure 1. Structure of Gd- ethoxybenzyl DTPA (Diethylenetriamine pentaacetate) (Eovist, Bayer Pharmaceuticals) that was used in the diffusion experiments here. At low pH in pineapple Eovist may lose some of the DTPA ligands, particularly in the presence of competing transition metal ions. 
Figure 2. Low energy $X$ ray image, from L-edge $X$-ray absorption by Gd3+, of slowly diffusing Eovist from an upper slice 2 hours apart (Top, A\&B) and the central slice 2 hours apart (Bottom, C\&D) of pineapple samples showing two locations as examples ( 1 and 2 O'clock positions, arrows) where Eovist was infused but seems to diffuse very slowly during the $2 \mathrm{hr}$ observation periods. By comparing these two slices, A\&C panels at the left with $B \& D$ at the right one can argue that Eovist structures are intact next to the rinds where $\mathrm{pH}$ may be high. However, at the core for the upper part of pineapple (image slice A at the top) Gd spreads within minutes presumably due to free Gadolinium with one or more DTPA ligands stripped. 


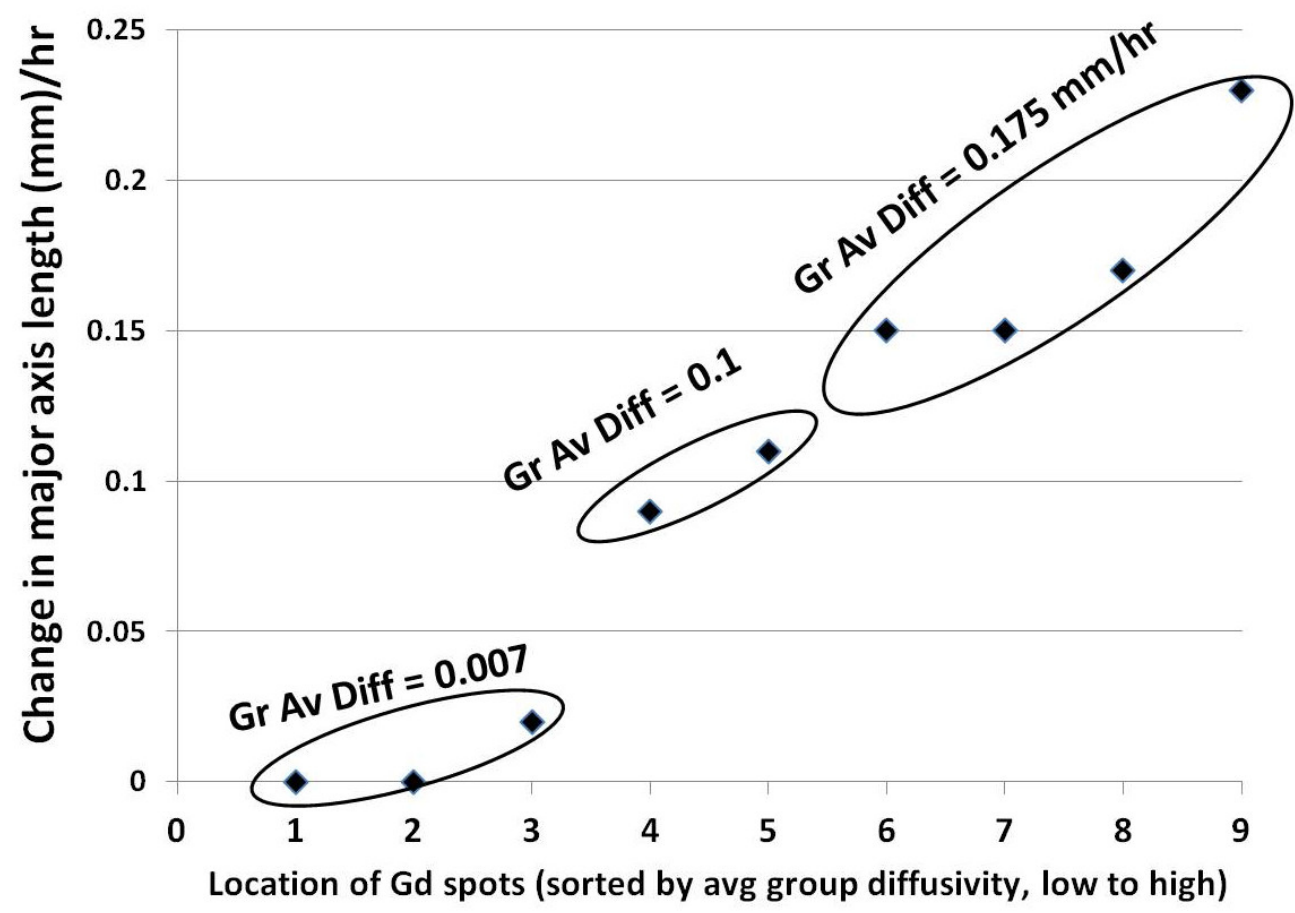

Figure 3 ( $A$, Left), 3 (B, Right). Measured diffusivities along the major and minor axes of elliptical Eovist spots are shown and grouped as slow, medium and fast groups (average diffusivities are noted in the plots, ranging an order of magnitude) presumably indicating transmetallation of $\mathrm{Gd} 3+$ with $\mathrm{Ca} 2+\mathrm{and} \mathrm{Mg}+2$ ions or other mechanisms to free Gd3+ ions that explains faster diffusion of Gd3+ with smaller inner sphere than the original Eovist. 


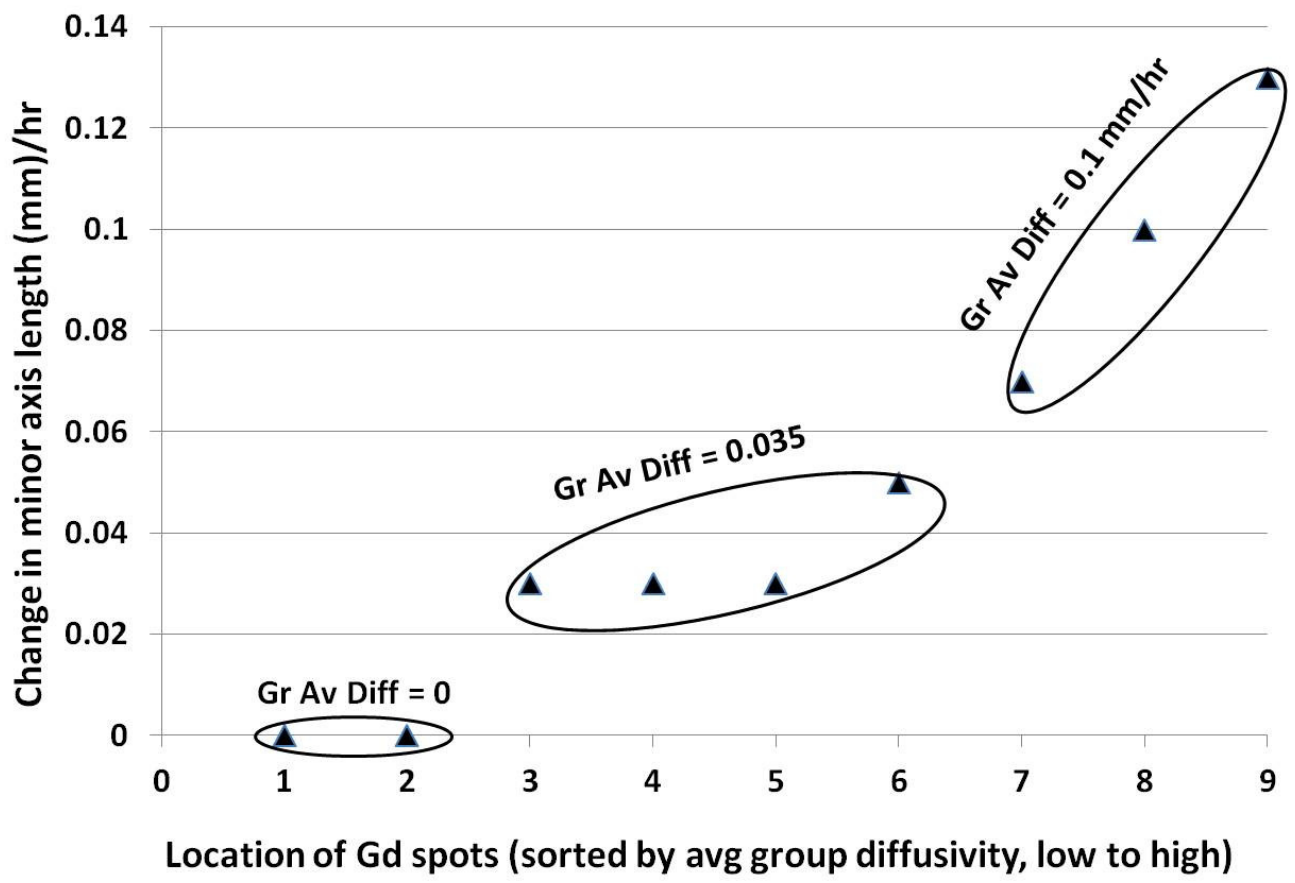

Figure 3 (A, Left), 3 (B, Right). Measured diffusivities along the major and minor axes of elliptical Eovist spots are shown and grouped as slow, medium and fast groups (average diffusivities are noted in the plots, ranging an order of magnitude) presumably indicating transmetallation of $\mathrm{Gd} 3+$ with $\mathrm{Ca} 2+$ and $\mathrm{Mg}+2$ ions or other mechanisms to free Gd3+ ions that explains faster diffusion of Gd3+ with smaller inner sphere than the original Eovist.

$103 \times 75 \mathrm{~mm}(300 \times 300 \mathrm{DPI})$ 


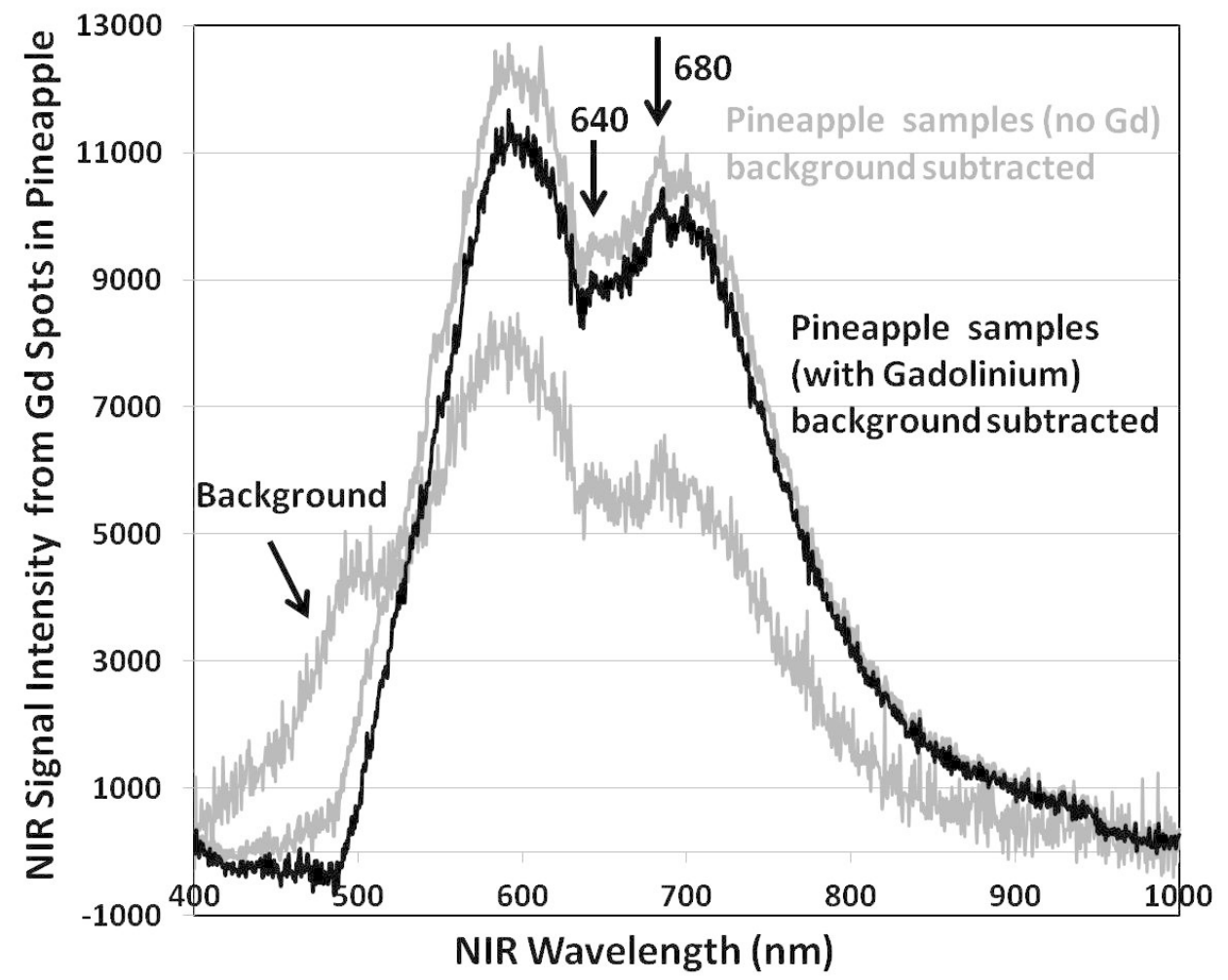

Figure 4. Near infrared background spectrum of the dark box (bottom trace, gray) that was subtracted from near infrared peaks of cut pineapple samples infused with Eovist (middle trace, black) and without Eovist (top trace, gray), Both the samples show near infrared induced photosynthesis peaks at 640 and $680 \mathrm{~nm}$ for pineapple systems, red-shifted by $30 \mathrm{~nm}$ compared to photosynthesis peaks (approximately 670 and 810

$\mathrm{nm}$ ) observed in cyano bacteria. [9]

$103 \times 82 \mathrm{~mm}(300 \times 300 \mathrm{DPI})$ 